\title{
Manejo conservador de los traumatismos renales de alto grado
}

\author{
Delgado Oliva FJ, Bonillo García MA, Gómez Pérez L, Oliver Amorós F, Gimeno Argente V, \\ Jiménez Cruz JF.
}

Servicio de Urología. Hospital La Fe. Valencia.

Actas Urol Esp. 2007;31(2):132-140

\begin{abstract}
RESUMEN
MANEJO CONSERVADOR DE LOS TRAUMATISMOS RENALES DE ALTO GRADO

Introducción: Los traumatismos genitourinarios constituyen el 8-10\% de los traumatismos abdominales y en el 50\% de los casos es el riñón el órgano más afectado, sobretodo el izquierdo. La decisión de adoptar un tratamiento conservador o quirúrgico en estos casos dependerá del tipo de lesión que encontremos en la unidad renal afecta y de las condiciones clínicas del paciente.

Objetivo: Los objetivos del estudio son determinar la posibilidad de llevar acabo un tratamiento conservador en los traumatismos renales de alto grado y estudiar la evaluación y aparición de complicaciones en los mismos.

Material y métodos: Hemos analizado retrospectivamente los 309 casos de traumatismos renales acontecidos en nuestro Servicio en el período de tiempo comprendido entre Enero 1984 y Enero 2006, estudiando variables como la etiología del traumatismo, lesiones asociadas en otros órganos, actitud terapéutica adoptada y presencia de complicaciones, tanto a largo como a corto plazo.

Resultados: De los 309 traumatismos renales analizados, el 94,1\% (291 casos) fueron traumatismos renales cerrados o contusos. La distribución por grados del total de traumatismos fue: Grado I: 213 casos (69\%), grado II: 39 casos (12.6\%), grados III y IV: 32 casos (10,3\%) y grado V: 25 casos (8\%). Realizamos tratamiento conservador en el 84,6\% de los casos etiquetados como grados III y grado IV ( 24 casos en total). Se practicaron 4 nefrectomías de urgencia en los grado III por inestabilidad hemodinámica y 4 nefrectomías en grados IV por el mismo motivo, una de ellas parcial, diferidas a las 4872 horas del traumatismo. En $67 \%$ de los traumatismos grado V se realizó nefrectomía de urgencia.

Conclusiones: De acuerdo a nuestra experiencia y a los resultados obtenidos, consideramos adecuado el manejo conservador en los traumatismos renales de alto grado siempre y cuando las condiciones hemodinámicas del paciente lo permitan.

Palabras clave: Traumatismo. Renal. Conservador.
\end{abstract}

\section{ABSTRACT}

\section{CONSERVATIVE APPROACH IN MAJOR RENAL TRAUMA}

Introduction: Genitourinary trauma amount to an 8-10\% of abdominal trauma with the kidney being the most affected organ in $50 \%$ of cases, especially the left one. The choice of treatment will depend on the kind of lesion found in the affected renal unit and on the patient's clinical conditions.

Objective: The aims of this study are twofold: to determine the applicability of conservative treatment in major renal trauma and to assess the evaluation and emergence of possible complications.

Material and methods: We have analysed 309 cases of renal trauma dealt with in our department between January 1984 and January 2006, analyzing such variables as the etiology of the trauma, associated lesions in other organs, the therapeutic approach adopted as well as the presence of complications, both in the long and short run.

Results: Out of a total of 309 renal trauma analyzed, a 94,1\% (291 cases) were blunt renal trauma. The distribution by grade was: Grade I, 213 cases (69\%); Grade II, 39 cases (12,6\%); Grade III/ IV, 32 cases (10,3\%); Grade V, 25 cases (8\%). We have given a conservative approach in the $84,6 \%$ of the grade III/ IV cases (24 cases). 4 grade III nephrectomies were carried out in the Emergency Room because of haemodynamic instability, other 4 grade IV nephrectomies were done for the same reason, one of which was a partial nephrectomy, 48-72 hours after the trauma. The treatment for grade V was nephrectomy in $67 \%$.

Conclusions: According to our experience and in the light of the results obtained, we consider the conservative approach adequate for major renal trauma as long as the patient is haemodynamically stable.

Keywords: Renal trauma. Conservative management. 
$\mathrm{L}$ os traumatismos son los responsables de la mayoría de los fallecimientos en pacientes menores de 40 años en los países desarrolla$\operatorname{dos}^{1,2}$. Dentro de las lesiones que encontramos en los pacientes politraumatizados, el traumatismo del aparato genitourinario acontece alrededor del 10\%4. De ellos, en el 50\% de los casos el riñón es el órgano más afectado, fundamentalmente el izquierdo ${ }^{3}$, siendo frecuentes las lesiones asociadas en otros órganos (20-94\% de los casos) $)^{5,14}$.

Destacar que en más del 90\% de los casos los traumatismos renales son contusos o cerrados ${ }^{6}$. Mención especial tienen aquellos casos de traumatismos renales contusos que se producen por desaceleración, muy frecuentes hoy día debido a los accidentes de tráfico, que generan un sobrestiramiento del pedículo renal con potencial lesión vascular del mismo ${ }^{7}$.

$\mathrm{El}$ avance en los métodos diagnósticos de imagen en el área de Urgencias, sobretodo la TAC ${ }^{8,9}$, supone poder realizar una evaluación más precisa y detallada de las lesiones renales y, si las condiciones hemodinámicas del paciente lo permiten, realizar un tratamiento conservador en estos casos, reduciendo de manera significativa el número de nefrectomías innecesarias ${ }^{10,11}$.

Uno de los puntos controvertidos en cuanto a la valoración terapéutica de los traumatismos renales es la variabilidad en las clasificaciones existentes, lo cual hace difícil comparar las distintas series publicadas, sobretodo con publicaciones americanas. Nosotros utilizamos la de Chatelain hasta 1995 y posteriormente la de la American Association for Surgery of Trauma (AAST) (Tabla 1).

Tabla 1

Clasificación de la AAST"

\begin{tabular}{ll}
\hline Grado & Hallazgos \\
\hline I & $\begin{array}{l}\text { Contusión renal (hematoma subcapsular) } \\
\text { II }\end{array}$ \\
& $\begin{array}{l}\text { Laceraciones menores (sin afectación medular } \\
\text { profunda ni de sistema colector) }\end{array}$ \\
III & Laceraciones mayores (urinoma) \\
IV & $\begin{array}{l}\text { Fractura renal } \\
\text { Lesiones vasculares aisladas }\end{array}$ \\
V & Avulsión pedicular/estallido renal \\
\hline
\end{tabular}

\section{MATERIAL Y MÉTODOS}

Hemos analizado retrospectivamente 309 casos de traumatismos renales valorados por el Servicio de Urología del Hospital La $\mathrm{Fe}$ de Valencia, en el periodo comprendido entre Enero 1984 y Enero 2006.

Las variables a estudiar fueron:

1. Etiología del traumatismo: valoraremos el mecanismo de producción del mismo (penetrantes/no penetrantes). Dentro de la etiología destacaremos aquellas que por frecuencia e importancia de las lesiones generadas nos vamos a encontrar en mayor número (accidentes de tráfico, heridas por arma blanca o arma de fuego y heridas por asta de toro).

2. Presencia de lesiones asociadas en otros órganos, con los elementos diagnósticos empleados (TAC, fundamentalmente, y ecografia / urografia en algunos de los casos).

3. Actitud terapéutica adoptada: quirúrgica o conservadora, según los hallazgos clínico / radiológicos que encontremos.

4. Presencia de complicaciones:

- A corto plazo: abcesos perirrenales, hematomas, urinomas.

- A largo plazo: fístulas, hipertensión postraumática (debida a fístulas arteriovenosas o a trombosis arterial).

Utilizaremos la clasificación de la AAST para cuantificar porcentualmente el número de lesiones presentes en cada caso, las complicaciones secundarias al traumatismo y el tratamiento practicado, observando la evolución en un periodo de seguimiento de entre 3-6 meses. También reflejaremos el número de nefrectomías realizadas y el motivo de las mismas.

También analizaremos otras variables como:

- Fecha de nacimiento y fecha del traumatismo.

- Antecedentes médicos y urológicos.

- Presentación clínica (hematuria, dolor y masa en flanco o hipotensión).

- Riñón afecto (derecho o izquierdo).

- Lesiones asociadas y tratamiento de las mismas (fracturas costales, con tratamiento médico/lesión del bazo con esplenectomía posterior).

- Estancia media hospitalaria (en días) y estancia en Unidad de Reanimación. 
- Necesidad de transfusión sanguínea (número de bolsas de concentrado de hematies).

- Tiempo de seguimiento en consulta externa hasta el alta definitiva

\section{RESULTADOS}

Los traumatismos cerrados o contusos que encontramos en nuestra serie supusieron el $94,1 \%$ de los casos. El riñón izquierdo aparece como el más frecuentemente lesionado en un $58 \%$ de los casos.

De los traumatismos renales abiertos descritos (18 casos), el 4,2\% (14 de los mismos) fueron consecuencia de herida por arma blanca y 4 por arma de fuego. En todos y cada uno de ellos se realizó exploración quirúrgica inmediata practicando nefrectomía en 4 casos (20\%).

El porcentaje de pacientes con antecedentes médicos y urológicos fue del 36.4\% y 5.6\%, respectivamente.

De acuerdo a esto último, en casi el $82 \%$ de los casos revisados se trataba de traumatismos grado I y II (69\% de los primeros frente al 12,6\% de los segundos), con un $8 \%$ de traumatismos grado $\mathrm{V}$.

En cuanto a la presentación clínica la serie revisada viene a confirmar que los hallazgos más frecuentes son la hematuria $(69,9 \%)$ y dolor en flanco afecto $(59,5 \%)$.

Con respecto a las lesiones asociadas hemos encontrado un $22 \%$ de fracturas costales y un $16 \%$ de lesiones esplénicas, que en vario casos (sobretodo en los traumatismos de mayor grado), precisaron de una actitud terapéutica más agresiva (drenaje torácico y esplenectomía) (Tabla 2).

En los traumatismos renales grados I y II el tratamiento, en casi el 100\% de los casos, fue conservador (Fig. 1). Sólo en 4 casos de los catalogados como grado II hubo que practicar nefrectomía de urgencia debido a la gravedad de las lesiones asociadas (afectación esplénica e intestinal) que obligaron a la realización de laparotomías exploradoras objetivando sangrado renal activo incoercible.

En cuanto a las complicaciones destacar en los grado II, el 22\% de casos con aumento del hematoma renal objetivado en TACs realizados, que se resolvieron espontáneamente, y el 11\% de muertes descritas debido a lesiones en otros órganos (Tablas 3 y 4).
Tabla 2

Variables generales de estudio

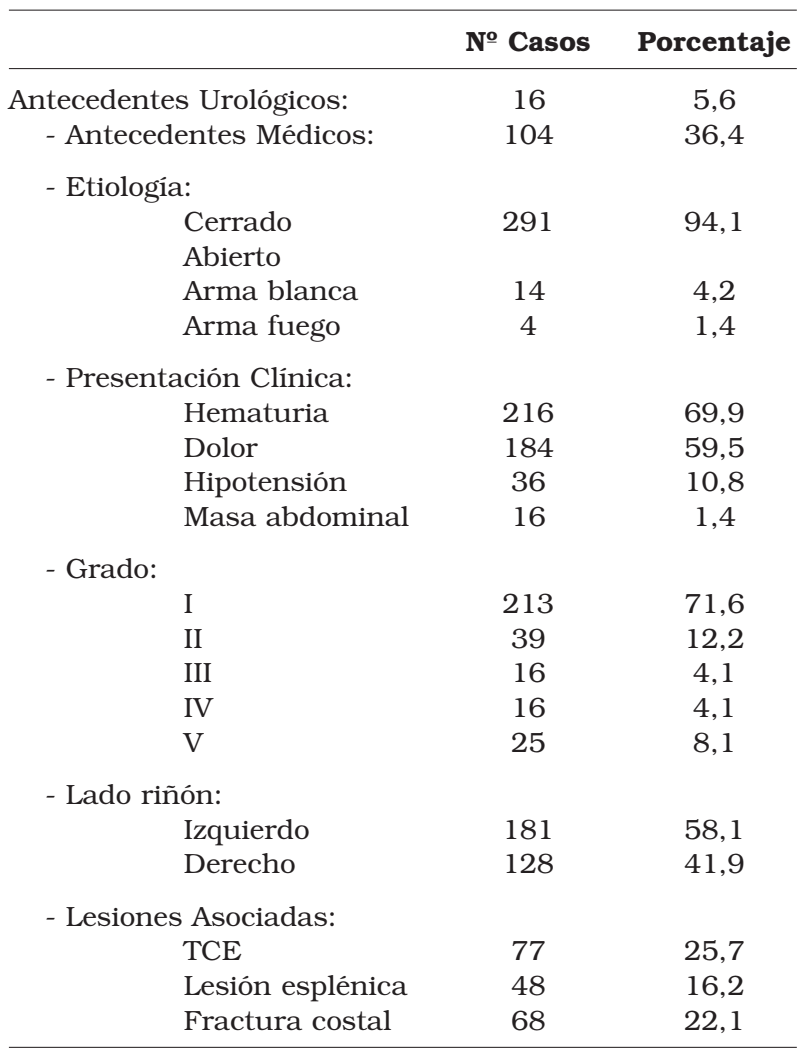

En los traumatismos grados III y IV optamos por el tratamiento conservador en el 84,6\% de los casos (Fig. 2). En los grado III se realizaron 4 nefrectomías de urgencia (en un 25\% de los casos) por inestabilidad hemodinámica; en estos casos también fue necesaria la esplenectomía.

Por otro lado, en los grado IV fueron necesarias 4 nefrectomías por fuga de contraste y shock hipovolémico posterior, aunque de forma diferida. En el 67\% de los casos de traumatismos renales grado IV que encontramos hubo que practicar esplenectomía.

No se describieron complicaciones en los grado III, mientras que en los grado IV en un 18\% (3 casos) se hallaron complicaciones como fiebre o urinoma que se resolvieron con tratamiento médico.

Mencionar la diferencia entre estos grados en cuanto a su estancia en Reanimación, ya que en los grado III ésta fue de 3 días y en los grado IV no fue necesaria hasta en el $67 \%$ de los casos. La media de unidades de concentrado de hematíes en los grado III fue de 3,6 (rango 3-4), frente a 8,8 (rango 6-12) en los de grado IV. 

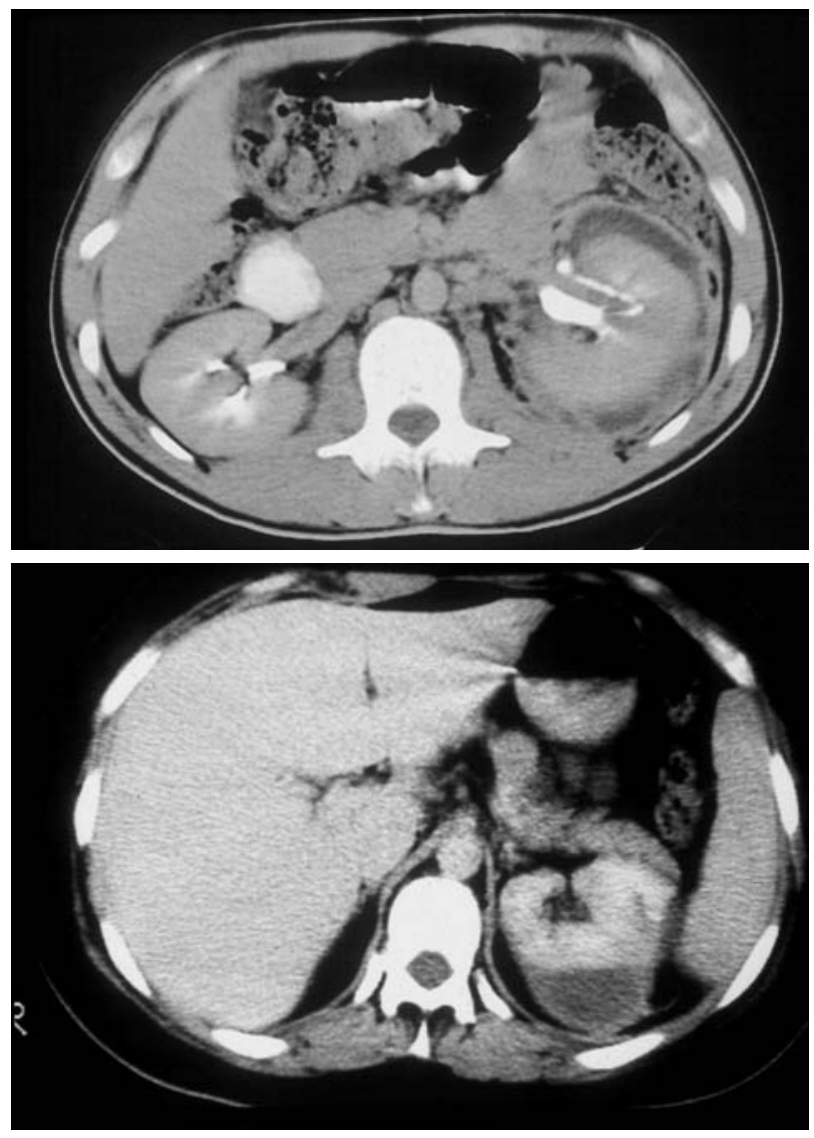

FIGURA 1. Traumatismos renales grado I y II.

Tabla 3

Traumatismos renales grado I

\begin{tabular}{lcc}
\hline Grado & No Casos & Porcentaje \\
\hline - Tto. Conservador: & 213 & 100 \\
- Otras cirugias: & & \\
$\quad$ Drenaje torácico & 4 & 1,9 \\
$\quad$ Esplenectomía & 8 & 3,8 \\
$\quad$ Estab. Óseas & 12 & 5,7 \\
- Complicaciones: & & \\
$\quad$ Fiebre & 28 & 13,2 \\
- Estancia media (días): & 9,57 & {$[3-29]$} \\
- Estancia REA (dias): & & \\
88,7\% No estancia & 0,58 & {$[0-15]$} \\
- UCH: & & \\
86,8\% No transfusión & 0,98 & {$[0-16]$} \\
- Seguimiento (meses): & & \\
62,3\% No seguimiento & 1,43 & {$[0-24]$} \\
\hline
\end{tabular}

Todos los casos de traumatismos grado IV fueron seguidos en consultas externas dados de alta sin necesidad de practicar procedimiento invasivo alguno. En los grado III no fue necesario el seguimiento durante más de 3 meses en el 67\% de los casos (Tablas 5 y 6).
Tabla 4

Traumatismos renales grado II

\begin{tabular}{|c|c|c|}
\hline Grado II & № Casos & Porcentaje \\
\hline - Tto. Conservador: & 35 & 89,7 \\
\hline - Nefrectomía urgencia: & 4 & 10,3 \\
\hline \multicolumn{3}{|l|}{ - Otras cirugias: } \\
\hline Drenaje torácico & 4 & 11,1 \\
\hline Esplenectomía & 4 & 11,1 \\
\hline Estab. Óseas & 4 & 11,1 \\
\hline Resección intestinal & 4 & 11,1 \\
\hline \multicolumn{3}{|l|}{ - Complicaciones: } \\
\hline $\begin{array}{l}\text { Aumento hematoma } \\
\text { Muerte }\end{array}$ & 8 & 22,2 \\
\hline Precoz (3h) & 4 & 11,1 \\
\hline Tardía (3d) & 4 & $\begin{array}{l}\text { Shock hipovol } \\
11,1 \\
\text { Fallo multiorg }\end{array}$ \\
\hline - Estancia media (días): & 19,11 & [1-103] \\
\hline - Estancia REA (días): & 2 & {$[0-5]$} \\
\hline - UCH: & 8,44 & [0-40] \\
\hline $\begin{array}{l}\text { - Seguimiento (meses): } \\
\text { 55,6\% No seguimiento }\end{array}$ & 2 & [0-6] \\
\hline
\end{tabular}

En los casos de traumatismos grado $\mathbf{V}$ el tratamiento predominante frente a la actitud conservadora fue la nefrectomía, de urgencia en el $67 \%$ de los casos y diferida (a las 4 horas) por shock hipovolémico en el 33\% de los mismos. En un porcentaje elevado de casos (84\%) fue necesaria la esplenectomía, dada la frecuencia e importancia de afectación multiorgánica en este tipo de traumatismos. También fueron relativamente frecuentes las complicaciones como fiebre, inestabilidad hemodinámica, IRA y muerte por fracaso multiorgánico (en la primera semana tras el traumatismo) en 4 casos (17\%).

En el 33\% de los casos se realizó tratamiento conservador como consecuencia de desgarros de la intima por sobreestiramiento de la arteria renal que pasan desapercibidos en el momento inminente del traumatismo por ausencia de clínica asociada (hematuria o dolor en fosa renal) (Fig. 3). Tras el desgarro la arteria se trombosa y el riñón acaba deteriorándose hasta la pérdida total del mismo, por tanto la práctica de la nefrectomía diferida tras la lesión intimal es innecesaria en estos casos, realizando seguimiento en consulta externa con vigilancia mediante ecografía o TAC (Tabla 7). 

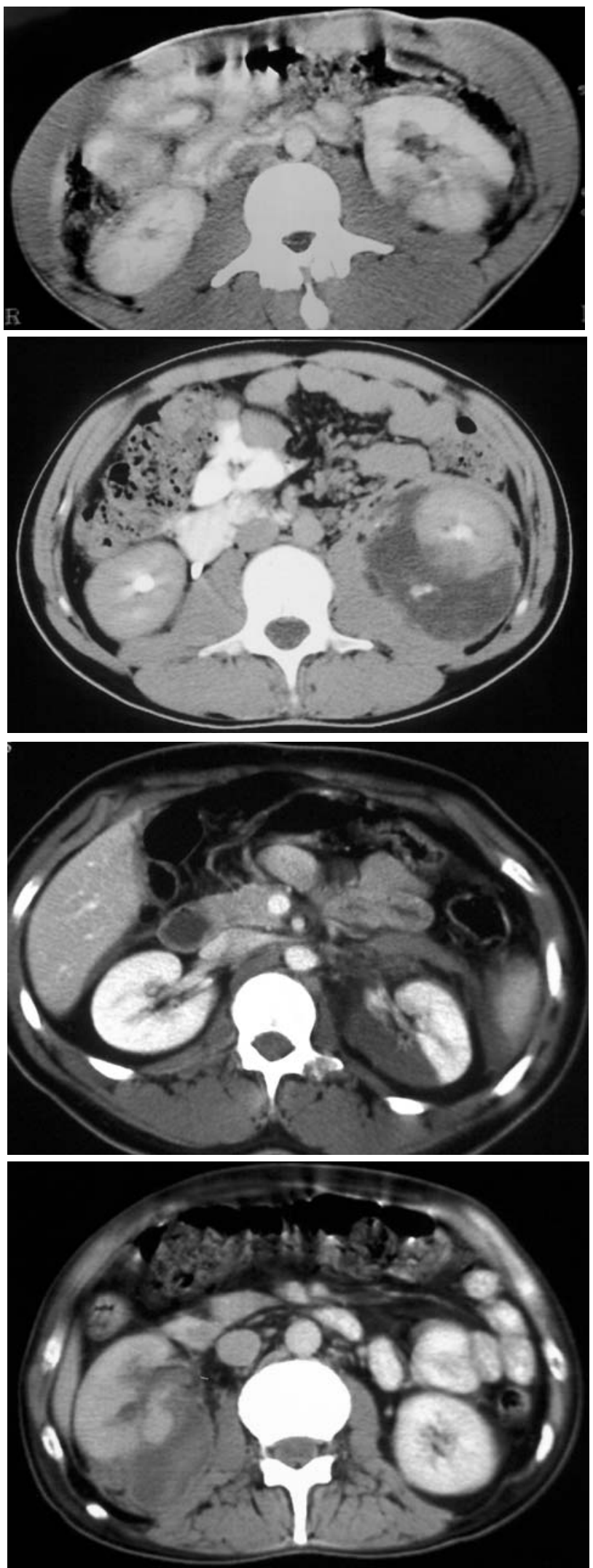

FIGURA 2. Traumatismos renales grado III y IV que se resolvieron con tratamiento conservador.
Tabla 5

Traumatismos renales grado III

\begin{tabular}{lcc}
\hline Grado III & No Casos & Porcentaje \\
\hline - Tto. Conservador: & 12 & 75 \\
- Nefrectomía urgencia: & 4 & 25 \\
- Otras cirugias: & & \\
$\quad$ Drenaje torácico & 4 & 33,3 \\
$\quad$ Esplenectomía & 4 & 33,3 \\
- Estancia media (días): & 18,67 & {$[12-30]$} \\
- Estancia REA (días): & 2,67 & {$[3-4]$} \\
- UCH: & 3,67 & {$[3-4]$} \\
- Seguimiento (meses): & & \\
66,7\% No seguimiento & 1,33 & {$[0-4]$} \\
\hline
\end{tabular}

Tabla 6

Traumatismos renales grado IV

\begin{tabular}{lcc}
\hline Grado & No Casos & Porcentaje \\
\hline $\begin{array}{l}\text { - Tto. Conservador } \\
\text { (de inicio) }\end{array}$ & 12 & 75 \\
- Nefrectomía parcial & & \\
diferida (24 h) & 1 & 25 \\
- Nefrectomía diferida & & Fuga contraste \\
(72 h) & 4 & 25 \\
$\quad$ & & Shock hipovol. \\
- Otras cirugias: & & 66,7 \\
$\quad$ Esplenectomía & 8 & 33,3 \\
$\quad$ - Complicaciones: & & 33,3 \\
$\quad$ Inestab. Hemo & 4 & 33,3 \\
$\quad$ Urinoma & 3 & {$[13-38]$} \\
$\quad$ & 30 & {$[0-2]$} \\
- Estancia media (días): & & {$[6-12]$} \\
- Estancia REA (días): & & \\
66,7\% No estancia & 0,70 & [2-4] \\
- UCH: & 8,87 & \\
- Seguimiento (meses): & 3 & \\
\hline
\end{tabular}

\section{DISCUSIŌN}

En los últimos años estamos asistiendo a un progresivo aumento en la gravedad y frecuencia de los traumatismos abdominales en general, y con ellos los genitourinarios, debido al gran número de accidentes de tráfico que se producen hoy día.

Uno de los puntos controvertidos en cuanto a la valoración terapéutica de los traumatismos renales es la variabilidad en las clasificaciones existentes, lo cual hace difícil comparar las distintas series publicadas, sobretodo con publicaciones americanas. Nosotros utilizamos la de la AAST, ya descrita anteriormente. 


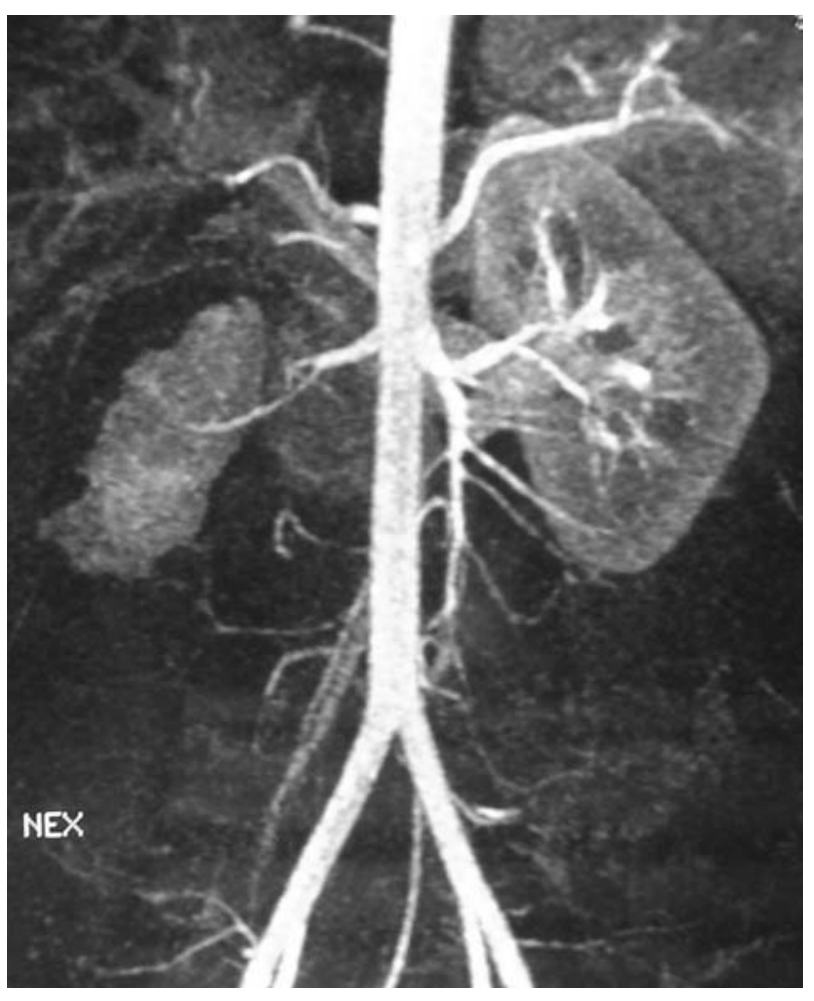

FIGURA 3. Traumatismo renal grado $\mathrm{V}$ con lesión vascular de la intima arterial, pérdida funcional de la unidad renal y atrofia parenquimatosa posterior.

Tabla 7

Traumatismos renales grado $\mathrm{V}$

\begin{tabular}{|c|c|c|}
\hline Grado & No Casos & Porcentaje \\
\hline - Tto. Conservador: & 8 & 33,3 \\
\hline - Nefrectomía urgencia: & 17 & 66,7 \\
\hline - Nefrectomía diferida (4h): & 8 & $\begin{array}{c}33,3 \\
\text { Shock hipovol }\end{array}$ \\
\hline \multicolumn{3}{|l|}{ - Otras cirugías: } \\
\hline $\begin{array}{l}\text { Drenaje torácico } \\
\text { Esplenectomía }\end{array}$ & $\begin{array}{c}8 \\
20\end{array}$ & $\begin{array}{l}33,3 \\
83,3\end{array}$ \\
\hline \multicolumn{3}{|l|}{ - Complicaciones: } \\
\hline Fiebre & 4 & 16,7 \\
\hline IRA leve & 4 & $\begin{array}{c}16,7 \\
\text { Diálisis }\end{array}$ \\
\hline Muerte (5 d) & 4 & $\begin{array}{l}16,7 \\
\text { Fallo multiorg. }\end{array}$ \\
\hline - Estancia media (días): & 27,33 & [4-94] \\
\hline - Estancia REA (días): & 9,87 & [0-30] \\
\hline - UCH: & 32,17 & [9-85] \\
\hline $\begin{array}{l}\text { - Seguimiento (meses): } \\
66,7 \% \quad \text { No seguimiento }\end{array}$ & 10,17 & {$[0-6]$} \\
\hline
\end{tabular}

En general, la injuria renal es secundaria a traumatismos no penetrantes o contusos, su extensión es limitada y no requiere cirugía. No obstante, el 5-10\% de las lesiones penetrantes y casi el $70 \%$ de las penetrantes son serias $^{12}$ y requieren una evaluación clínica y radiológica cuidadosa.

La presentación clínica del los traumatismos renales es variada, pero generalmente cursan con hematuria (69\% en nuestra serie), dolor en flanco afecto, (58\%), inestabilidad hemodinámica (hipotensión arterial brusca) y/o masa palpable ( $1,4 \%$, en nuestro estudio). Destacar que siendo la hematuria el rasgo distintivo de las lesiones renales, su grado no se correlaciona con la extensión de la lesión ${ }^{25}$. Además, en pacientes con lesiones renales vasculares, la hematuria puede no estar presente en el 19-40\% de los casos, dificultando el diagnóstico de las mismas ${ }^{26}$.

Hasta hace pocas décadas la nefrectomía hemostática o la nefrectomía diferida por secuelas graves eran las únicas indicaciones quirúrgicas en los traumatismos renales. De estas premisas tan simples hemos pasado a realizar tratamientos más complejos y conservadores con la finalidad de mantener el máximo parénquima renal funcionante ${ }^{14}$.

En los últimos años el algoritmo diagnóstico de estas lesiones ha cambiado sustancialmente con el uso de la ecografia y la TAC ${ }^{15,20}$, incluso en el área de Urgencias, a diferencia de las exploraciones que se utilizaban hace pocos años como la urografía y la arteriografía. La urografía ha pasado a tomar un lugar secundario en la evaluación de los traumatismos renales, limitada generalmente al estudio diferido en Consulta Externa. La arteriografía, por otro lado, juega un papel muy restringido ante la sospecha de lesión del pedículo, trombosis de la arteria renal y fístula arteriovenosa y, dada la no disponibilidad para su uso en Urgencias, ha sido desplazada por la TAC ${ }^{14}$; según algunos autores ésta última podría definir lesiones pediculares ${ }^{8,13}$.

Como estamos comentando la exploración diagnóstica que ha demostrado mayor utilidad en el área de Urgencias es la TAC, por ser rápida, incruenta y estar disponible en la gran mayoría de hospitales. Establece con exactitud el grado de la lesión, revelando desgarros vasculares y hemorragias circundantes, laceraciones y fracturas parenquimatosas y la presencia de lesiones asociadas en otros órganos. Toda esta información que nos aporta la TAC posibilita el tratamiento 
conservador en casos seleccionados, confirmando el cambio de actitud en el diagnóstico y tratamiento de este tipo de pacientes ${ }^{14}$.

Si bien el uso de la ecografia y la TAC se han establecido hoy día como las exploraciones diagnósticas fundamentales en la valoración del trauma renal ${ }^{15}$, aún existe polémica sobre el tratamiento a seguir en los mismos. En las lesiones extremas, es decir, los grados I / II y grado V, el tratamiento parece claro, conservador en los primeros y nefrectomía de urgencia en los segundos. La excepción a esto último en cuanto los traumatismos renales grado $\mathrm{V}$ son aquellos casos de desgarros de la íntima que pasan desapercibidos clínicamente en el momento del traumatismo con trombosis renal y pérdida funcional total del órgano, siendo innecesaria la nefrectomía ${ }^{13}$; en nuestra serie esto ocurrió en 8 de los 25 casos revisados de traumatismos grado $\mathrm{V}$.

La mayor polémica se mantiene cuando hablamos del tratamiento de los traumatismos renales grado III y IV. Como ya hemos comentado, estamos asistiendo a un cambio sustancial en el enfoque diagnóstico y terapéutico de este tipo de lesiones en los últimos años ${ }^{16,17}$, ya que hace poco más de una década la gran mayoría de los autores se decantaban por mostrar una actitud intervencionista (quirúrgica), a diferencia de aquellos que muestran tendencia a un manejo conservador (médico), como ocurre actualmente en la gran mayoría de las series publicadas.

Los defensores del tratamiento quirúrgico inmediato ${ }^{21,22}$ aducen que el tratamiento conservador se asocia a una elevada incidencia de hemorragias secundarias. Además el urohematoma puede infectarse dando lugar a la formación de abcesos u organizarse produciendo la compresión de la vía urinaria con ureterohidronefrosis secundaria, atrofia renal o hipertensión arterial, incrementándose pues la tasa de complicaciones tanto precoces como tardías.

Por el contrario, los que abogan por la práctica de un tratamiento médico ${ }^{1,10,23}$ parecen demostrar en sus series que el porcentaje de nefrectomías es mayor cuando se interviene en los días inmediatos al traumatismo, debido al riesgo de hemorragia brusca e incontrolable que obliga a extirpar la unidad renal. Estos autores también afirman que casi todas las extravasacio- nes pequeñas y muchas de las mayores cierran espontáneamente, reabsorbiéndose asimismo el hematoma en unas semanas.

Junto a ello, el perfeccionamiento de las técnicas percutáneas, bajo control ecográfico o radiológico, permite evacuar hematomas y urinomas que comprometan la permeabilidad de la vía urinaria o que amenacen la recuperación de la función renal, facilitando el tratamiento conservador $\mathrm{y}$ evitando nefrectomías innecesarias $^{18,19,20}$. En nuestra serie no fue necesario evacuar de forma percutánea ninguna extravasación urinaria y manejamos el urinoma traumático mediante colocación de catéter doble $\mathrm{J}$ y sonda vesical para mantener en reposo la vejiga y evitar así el reflujo vesicoureteral. La sonda la retiramos cuando las condiciones hemodinámicas del paciente son adecuadas y la hematuria se ha resuelto y el catéter a las 6-8 semanas.

Tampoco podemos olvidar el hecho de que en la intervención inmediata se extirpa con seguridad tejido recuperable. Pasado el momento inmediato al traumatismo y a la vista de la evolución, puede decidirse una cirugía diferida, considerándose como tiempo óptimo entre el $3^{\circ}$ y $10 \stackrel{\circ}{ }$ día $^{24}$, ya que en estos casos la cirugía suele ser conservadora y más sencilla estando delimitadas las áreas isquémicas y la hemostasia se ha producido espontáneamente .

Nosotros optamos por un tratamiento conservador en el $84,6 \%$ de los traumatismos renales grado III y IV. Realizamos nefrectomía de urgencia en 4 de los 16 casos (25\%) de traumas grado III por inestabilidad hemodinámica (en estos casos también fue necesaria la esplenectomía). En aquellas lesiones etiquetadas como grado IV se practicó nefrectomía parcial diferida (a las 24 h) en 1 caso por fuga de contraste objetivada en la TAC y nefrectomía total en 4 casos a las 72 horas por shock hipovolémico.

En aquellos traumas renales grado III que fueron tratados con medidas conservadoras no objetivamos complicación alguna ni a corto (en forma de abcesos y/o urinomas), ni a largo plazo (fístulas o hipertensión arterial postraumática). Igualmente ocurrió en los grado IV, en los que no constatamos complicaciones tardías tras el tratamiento expectante. Sin embargo, en éstos últimos se describieron complicaciones inmediatas como 
la fiebre o fuga de contraste en forma de urinoma (3 casos), que se resolvieron con tratamiento médico. En este caso el urinoma se trató con antibioterapia de amplio espectro y colocación de sonda vesical y de un catéter doble $\mathrm{J}$ que se retiró a las 6-8 semanas.

\section{CONCLUSIONES}

Asistimos en los últimos años a un cambio importante en el abordaje diagnóstico-terapéutico de los traumatismos renales. Éstos, a su vez, están aumentando cada vez más debido a la alta tasa de accidentes de tráfico que hoy día tenemos que atender en los servicios hospitalarios. Por ello, creemos fundamental intentar estandarizar y protocolizar las medidas a tomar ante los mismos. Sin embargo, el tipo de trauma, grado de injuria renal y extensión de las lesiones asociadas varía según las series consultadas, haciendo difícil la comparación entre ellas.

En base a nuestra experiencia y a la vista de los resultados obtenidos, pensamos adecuado e indicado el tratamiento conservador en los traumatismos renales grados III y IV, debido a que de esta manera se reduce significativamente el porcentaje de nefrectomías innecesarias y al escaso porcentaje de complicaciones (inmediatas y tardías) asociadas.

También pensamos que en caso de necesitar cirugía conservadora diferida el periodo ideal para llevarla a cabo es el comprendido entre el $3^{\circ}$ y $10^{\circ}$ día post traumatismo.

No obstante creemos lógico asumir que cada caso debe ser considerado de forma individualizada, teniendo en cuenta el tipo de lesión, hallazgos radiológicos y situación hemodinámica del paciente a la hora de decantarse por una $u$ otra actitud terapéutica.

\section{REFERENCIAS}

1. Wessells H, Suh D, Porter JR, Rivara F, MacKenzie EJ, Jurkovich GJ, et al. Renal injury and operative management in the United States: results of a population-based study. J Trauma. 2003;54(3):423-430.

2. Altman L, Haas C, Dinchman KH, Spirnak JP. Selective nonoperative management of blunt grade 5 renal injury. J Urol. 2000 Jun;164(1):2730 .

3. Napal Lecumberri S, Pascual Piedrola I, Solchaga Martinez A, Arrondo Arrondo JL, Ipiens Aznar A. Traumatismos renales: Revisión de 149 casos. Cambio en la actitud diagnóstica y terapeútica. Arch Esp Urol. 1992;45(4):305-315.

4. Burger RA, Hohenfellner T. Urology Trauma. Pediatr Nephrol. 1989;3(2): 209-212.

5. McAninch JW, Carrol PR, Armenakas NA. Renal gunshot wounds: methods of salvage and reconstruction. J Trauma.1993;35(2):279-283.
6. Vicente J. Pauta de actuación. Traumatismos genitourinarios. En: Pautas de actuación y protocolos asistenciales. Pulso Ed., Barcelona. España, 1995;45-51.

7. Schmidlin F, Farshad M, Bidaut L, Barbezat M, Becker C, Niederer P et al. Biomechanical analisis and medical treatment of blunt renal trauma. Swiss Surg. 1998;5:237-243.

8. Bretan P, McAninch JW, Federle MP, Jeffrey RB. Computerized tomographic stagins of renal trauma: 85 consecutives cases. J Urol. 1986;136(3):561-565.

9. Herschorn S, Radomski SB, Shoskes DA, Mahoney J, Hirshberg E, Klotz L. Evaluation and treatment of blunt renal trauma. J Urol. 1991;146(2):274-277.

10. Danuser H, Wille S, Zöscher G, Studer UE. How to treat blunt kidney ruptures: Primary open surgery or conservative treatment with deferred surgery when necessary?. Eur Urol. 2001;39(1):9-14.

11. Kawashima A, Sandler C, Corl F, West OC, Tamm EP, Fishman EK et al. Imaging evaluation of posttraumatic renal injuries. Abdom Imaging. 2002;27(2):199-213.

12. Carrol PR, McAninch JW. Current management of blunt renal trauma. Urol Annu 1987;1:171-176.

13. Wessells H, McAninch JW. Actualización sobre los traumatismos de las vias urinarias superiores. AUA Updates Series 1997.

14. Santucci RA, Wessells H, Bartsch G, Descotes J, Heynes, CF, McAninch JW, Nash, P, Schmidlin F. Evaluation and management of renal injuries: consensus statement of the renal trauma subcommittee. BJU Int. 2004;93(7):937-954.

15. McGahan JP, Richards S, Jones CD, Gerscovich EO. Use of ultrasonography in the patient with acute renal trauma. J Ultrasound Med. 1999;18(3):207-216.

16. Begqvuist D, Hedelin H, Lindblad B. Blunt renal trauma. Changes in etiology, diagnostic procedure, treatment and complications over thirty years. Scand J Urol Nephrol. 1980;14(2):177-180.

17. Carmona Campos EC, Prieto Castro R, Regueiro López JC, Álvarez Kindelan J, Gomez Bermudo J, Anglada Curado F et al. Evaluación y tratamiento del traumatismo renal cerrado. Actas Urol Esp.1998; 22(1):23-28.

18. Morano Ju, Burkhalter JL. Percutaneous catheter drainag of postraumatic urinoma. J Urol. 1985;134:319-321.

19. Wilkinson AG, Haddock G, Carachi R. Separations of renal fragments by a urinoma after renal trauma: percutaneous drainage acclerates healing. Pediatr Radiol. 1999;29(7): 503-505.

20. Heyns CF. Renal trauma: indications for imagin and surgical exploration. BJU Int. 2004;93(8):1165-1170.

21. Cass AS, Luxenberg M, Gleich P, Smith C. Long-term results of conservative and surgical management of blunt renal lacerations. BJU Int. 1987;59(1):17-20.

22. Kristjansson A, Pedersen J. Management of blunt renal trauma.. Br J Urol. 1993 Nov;72(5 Pt 2):692-696

23. Moudouni SM, Patard JJ, Manunta A, Guiraud P, Guille F, Lobel B. A conservative approach to major blunt renal lacerations with urinary extravasation and devitalized renal segments. BJU Int. 2001;87(4): 290-294.

24. Montserrat Monfort JJ, Oliver Amoros F, Arlandis Guzmán S, Pontones JL, Rodrigo Aliaga JL, López Alcina E et al. Traumatismos renales: protocolo diagnóstico e indicaciones terapéuticas. Actas Urol Esp.1996;20(6):534-543.

25. Bright TC, White K, Peters PC. Significance of hematuria after trauma. J Urol. 1978;120(4):455-456.

26. Cass AS. Renovascular injuries from external trauma: Diagnosis, treatment and outcome. Urol Clinic N Amer. 1989;16(2):213-220.

Correspondencia autor: Dr. F.J. Delgado Oliva.

Servicio Urología. Hospital Universitario La Fe.

Avda. Campanar, 21. 46009 Valencia. Tel.: 963862700

E-mail autor: frandelgol@hotmail.com

Información artículo: Original - Traumatismos

Trabajo recibido: septiembre 2006

Trabajo aceptado: octubre 2006 


\section{COMENTARIO EDITORIAL}

Los autores presentan una importante serie de traumatismos renales recogidos en su medio hospitalario durante 2 décadas, donde predominan los traumatismos de bajo grado en concordancia con otras series ya publicadas. Generalmente son los traumatismos grados III y IV los que nos plantearán la duda de mantener una actitud conservadora o agresiva, que en ausencia de cambios radiológico y hemodinámicos como indican los autores ofrecerá la oportunidad para la estabilización clínica y la conservación renal. En nuestra experiencia ${ }^{1}$ comparando una serie de 188 traumatismos renales en un período de dos décadas, la proporción de nefrectomías en la $1^{\underline{a}}$ década fue más del doble de las realizadas en la $2^{\underline{a}}$ década $(\mathrm{p}<0,001)$ para el mismo grado de lesión. Finalmente es de agradecer a los autores que incluyan en su revisión trabajos nacionales muchas veces ignorados.

\section{REFERENCIAS}

1. Ipiens A, Solchaga A, Pascual JI. Traumatismos renales. En Tratado de Urología, J.F. Jiménez Cruz y L.A. Rioja Sanz, editores. En prensa, 2006.

Fdo.: J. Ignacio Pascual

\section{RESPUESTA AL COMENTARIO EDITORIAL}

En nuestra serie predominan los traumatismos renales de bajo grado, porque como se menciona en gran parte de la bibliografía consultada ${ }^{1-}$ ${ }^{3}$, más del $90 \%$ de los traumatismos renales que tenemos quer atender en los Servicios Hospitalarios son contusos o cerrados. En estas circunstancias, en 22 años de periodo de estudio, hemos objetivado "32 casos (10\%) de traumatismos renales grados III y IV y 25 casos (8\%) de traumas grados V. Pensamos que es un número considerable dentro de las series nacionales que hemos revisado. De todas formas, estaríamos interesados que los compañeros nos enviaran la referencia exacta dónde se analizan los 188 traumatismos que nos comentan para estudiarla y referenciarla en nuestro trabajo. Muchas gracias.

\section{REFERENCIAS}

1. Vicente J: Pauta de actuación. Traumatismos genitourinarios. En: Pautas de actuación y protocolos asistenciales. Pulso Ed., Barcelona. España, 1995;45-51.

2. Santucci RA, Wessells H, Bartsch G, Descotes J, Heyns CF, McAninch JW et al. Evaluation and management of renal injuries: consensus statement of the renal trauma subcommittee. BJU Int 2004;93(7):937-954.

3. Heyns CF. Renal trauma: indications for imagin and surgical exploration. BJU Int 2004;93:1165-1170.

Fdo.: F.J. Delgado Oliva 